Research Article

Animal Genetics

\title{
Cytotaxonomy of Gallinula melanops (Gruiformes, Rallidae): Karyotype evolution and phylogenetic inference
}

Ivanete de Oliveira Furo ${ }^{1,2}$ (10), Rafael Kretschmer ${ }^{3,4}$ (D), Patricia C. M. O’Brien², Jorge Claudio da Costa Pereira ${ }^{2,5}$, Ricardo José Gunski ${ }^{6}$ (D), Analía Del Valle Garnero ${ }^{6}$ (D), Rebecca E. O'Connor ${ }^{4}$ (D), Darren Karl Griffin ${ }^{4}$, Malcolm A. Ferguson-Smith ${ }^{2}$ and Edivaldo Herculano Corrêa de Oliveira ${ }^{7,8}$ (D)

\author{
${ }^{1}$ Universidade Federal Rural da Amazônia (UFRA) Laboratório de Reprodução Animal (LABRAC), \\ Parauapebas, PA, Brazil. \\ ${ }^{2}$ University of Cambridge Department of Veterinary Medicine, Cambridge Resource Centre \\ for Comparative Genomics, Cambridge, United Kingdom. \\ ${ }^{3}$ Universidade Federal do Rio Grande do Sul, Programa de Pós-graduação em Genética e Biologia \\ Molecular (PPGBM), Porto Alegre, RS, Brazil. \\ ${ }^{4}$ University of Kent, School of Biosciences, Canterbury, United Kingdom. \\ ${ }^{5}$ University of Trás-os-Montes and Alto Douro (UTAD), Animal and Veterinary Research Centre (CECAV), \\ Vila Real, Portugal. \\ ${ }^{6}$ Universidade Federal do Pampa, Programa de Pós-graduação em Ciências Biológicas (PPGCB), São \\ Gabriel, RS, Brazil. \\ ${ }^{7}$ Instituto Evandro Chagas, Laboratório de Cultura de Tecidos e Citogenética (SAMAM), Ananindeua, \\ PA, Brazil. \\ ${ }^{8}$ Universidade Federal do Pará, Instituto de Ciências Exatas e Naturais, Belém, PA, Brazil.
}

\begin{abstract}
Although Rallidae is the most diverse family within Gruiformes, there is little information concerning the karyotype of the species in this group. In fact, Gallinula melanops, a species of Rallidae found in Brazil, is among the few species studied cytogenetically, but only with conventional staining and repetitive DNA mapping, showing $2 n=80$. Thus, in order to understand the karyotypic evolution and phylogeny of this group, the present study aimed to analyze the karyotype of G. melanops by classical and molecular cytogenetics, comparing the results with other species of Gruiformes. The results show that G. melanops has the same chromosome rearrangements as described in Gallinula chloropus (Clade Fulica), including fission of ancestral chromosomes 4 and 5 of Gallus gallus (GGA), beyond the fusion between two of segments resultants of the GGA4/GGA5, also fusions between the chromosomes GGA6/GGA7. Thus, despite the fact that some authors have suggested the inclusion of $G$. melanops in genus Porphyriops, our molecular cytogenetic results confirm its place in the Gallinula genus.
\end{abstract}

Keywords: Birds, Clade Fulica, chromosome painting, chromosome evolution, microchromosomes.

Received: July 14, 2020; Accepted: January 29, 2021.

\section{Introduction}

Gruiformes is an avian order showing great heterogeneity of habits, habitats and morphology and a wide geographic distribution (Del Hoyo et al., 1996; Garcia et al., 2014). Because of their great diversity, phylogenetic relationships among the different families in this order are still controversial, despite the number of phylogenetic studies performed so far. One of the first proposals based on morphological characters classified Gruiformes into 12 families (Wetmore, 1960). However, with the introduction of new methods, such as molecular studies using mitochondrial-nuclear DNA or genome sequencing, it has been possible to reach a consensus

Send correspondence to Edivaldo H. C. de Oliveira. Instituto Evandro Chagas, Laboratório de Cultura de Tecidos e Citogenética (SAMAM), 67030-000, Ananindeua, PA, Brazil. Email: ehco@ ufpa.br. that there is a monophyletic core of five families known as "Core Gruiformes":Rallidae, Heliornithidae, Psophiidae, Aramidae, and Gruidae (Fain et al., 2007; Hackett et al., 2008; Jarvis et al., 2014; Prum et al., 2015).

Within the core Gruiformes, Rallidae is the family with the highest number of species, around 152, distributed in 33 to 40 genera, comprising $85 \%$ of the order diversity (Garcia et al., 2014; Gill et al., 2020). The phylogenetic relationships within Rallidae still present many inconsistencies, due mainly to the small numbers of species that have been sampled in the different approaches (Garcia et al., 2014).

Rallidae shows a huge geographic distribution and taxonomic complexity (Livezey, 1998). Several species of this family, including the Spot-flanked Gallinule (Gallinula melanops), are distributed from northeastern to southern Brazil, with occurrences in Bolivia, Paraguay and Argentina southward (Taylor, 1996; Taylor and van Perlo, 1998). G. melanops $(2 \mathrm{n}=80)$ is one of the six Rallidae species 
with known karyotypes, together with Fulica atra $(2 \mathrm{n}=92)$, Gallinula chloropus $(2 \mathrm{n}=78)$, Aramides cajaneus $(2 \mathrm{n}=78)$, Porzana albicollis $(2 \mathrm{n}=72)$, and Porphyrio porphyrio $(2 \mathrm{n}=80)$ (Giannoni and Giannoni, 1983; Hassan, 1998; Nanda et al., 2011; Gunski et al., 2019; Furo et al., 2020). Hence, in the classification proposed by Garcia et al. (2014), only species belonging to clade Fulica (genera Fulica, Gallinula and Porzana), Aramides (Aramides and Porzana albicollis) and Porphyrio (genus Porphyrio) have been analyzed by classical or molecular cytogenetics.

The introduction of new cytogenetic tools, especially comparative chromosome painting has helped to improve the understanding of karyotype evolution and phylogenetic relationships among different species of birds (Kretschmer et al., 2014, 2015, 2018a, 2020; Furo et al., 2015, 2018; Rodrigues et al., 2018). The variety of whole chromosome painting probes now available include chicken (Gallus gallus-GGA), stone-curlew (Burhinus oedicnemus-BOE), white hawk (Leucopternis albicollis-LAL), griffon vulture (Gyps fulvus-GFU) and eared dove (Zenaida auriculataZAU) (Nie et al., 2009; de Oliveira et al., 2010; Kretschmer et al., 2018b). Moreover, the use of bacterial artificial chromosomes (BACs) from the genome library of $G$. gallus has overcome much of the difficulty in the analysis of microchromosome rearrangements (Lithgow et al., 2014; O'Connor et al., 2019).

There is an urgent need to use these new techniques to clarify the problems concerning avian karyotypes and phylogenetic relationships in a greater number of species (Dobigny et al., 2004; Furo et al., 2015, 2018; Nie et al., 2015; Seligmann et al., 2019). The main aim of this study was to characterize the karyotype of $G$. melanops by classic cytogenetics, GGA chromosome painting probes and FISH with BACs selected from the genome library from microchromosomes of G. gallus in order to contribute to the phylogeny and karyotype evolution of the Rallidae family.

\section{Material and Methods}

\section{Chromosome preparation}

Fibroblast cultures obtained from wing skin biopsies of five female specimens of Gallinula melanops were collected in São Gabriel, Rio Grande do Sul State, (RS, Brazil), following Sasaki et al. (1968) with modifications. The samples were first mechanically fractionated in a Petri dish after incubation in type IV collagenase for tissue dissociation. The cells were cultured in DMEM (GIBCO) supplement with calf bovine serum 20\%, Aminiomax ${ }^{\mathrm{TM}}-$ II 5\% and penicillin (PNS) $1 \%$ then incubated at $37{ }^{\circ} \mathrm{C}$. Afterwards, metaphase arrest was obtained by adding colcemid (Gibco, $100 \mu 1$ for $5 \mathrm{ml}$ of complete medium) followed by incubation for 1 hour at $37^{\circ} \mathrm{C}$, and hypotonic solution treatment $(\mathrm{KCl}$ $0,075 \mathrm{M}$ ) for 15 minutes. Finally, the suspensions were fixed using Carnoy's fixative methanol: acetic acid (3:1 $\mathrm{v} / \mathrm{v})$. The experiments followed ethical protocols approved by the Ethics Committee $n^{\circ} 018 / 2014$ (UNIPAMPA) and SISBIO: 44173-3.

\section{Fluorescence in situ Hybridization (FISH)}

Gallus gallus (GGA) chromosome probes from 1 to 14 obtained by flow-sorting and labeled with biotin-16-dUTP or digoxigenin-11-dUTP (Roche Diagnostics, Mannheim, Germany) by degenerate oligonucleotide-primed polymerase chain reaction (DOP-PCR) (Telenius et al., 1992) were hybridized to metaphase chromosomes of G. melanops, following standard protocols, as described previously by de Oliveira et al. (2010). In this study ZW chromosome probes of G. gallus were not used. The FISH results were analyzed using a Zeiss Imager 2 microscope, $63 \mathrm{x}$ objective and images were captured using Axiovision 4.8 software (Zeiss, Germany). At least 10 metaphases were analyzed to confirm the hybridizations signals. Final editing of images was performed using Adobe Photoshop CC software. For chromosomal evolution inferences, we used chromosome painting data from Fulica atra, Gallinula chloropus and Aramides cajaneus (Nanda et al., 2011; Furo et al., 2020), also these data were plotted in a phylogenetic tree proposed by Garcia et al., (2014), to clarify the phylogenetic position of some Rallidae species.

The bacterial artificial chromosomes (BACs) were selected from the genome library of G. gallus or Taeniopygia guttata (Zebra finch) for the microchromosomes GGA17-28, following O'Connor et al. (2019). Slides were analyzed with an Olympus BX-61 epifluorescence microscope equipped with a cooled CCD camera and appropriate filters. Images were captured using SmartCapture3 (Digital scientific UK).

\section{Results}

\section{Karyotype and chromosome painting with G. gallus probes}

The karyotype of Gallinula melanops $(2 \mathrm{n}=80)$ is composed of 11 pairs of macrochromosomes, including the $\mathrm{Z}$ and $\mathrm{W}$, and 29 pairs of microchromosomes, which corroborates previous findings (Gunski et al., 2019). The first and second chromosome pairs are submetacentrics, the third, fourth and fifth pairs are metacentrics and the other chromosome are telocentrics. The sex chromosomes ZW are submetacentrics, and the $\mathrm{W}$ is larger than the $\mathrm{Z}$ chromosome due to the accumulation of repetitive DNA, as described by Gunski et al. (2019) (Figure 1).

The hybridization of fluorescent whole chromosome probes from $G$. gallus to the metaphase chromosomes of G. melanops shows fission events involving of ancestral cromosomes GGA4 and GGA5, beyond the fusion between two of segments resultants of the GGA4 and GGA5 (Figure 2D). Also, fusions were observed between chromosomes GGA6 and GGA7. The chromosome correspondences were: GGA1GME1; GGA2- GME2; GGA3- GME3; GGA4- GME4p, GME7 and GME13; GGA5- GME4q and GME12; GGA6GME5p; GGA7- GME5q; GGA8- GME6; GGA9- GME8; GGA10-12- GME9 and GME10; GGA13- GME14; GGA14GME15 (Table 1 and Figures 2 and 4). Each chicken or Zebra finch BAC probe from microchromosomes 17 to 28 hybridized only to one pair of microchromosomes, revealing that they were conserved and did not participate in interchromosomal rearrangements (Table 2, Figure 3). 


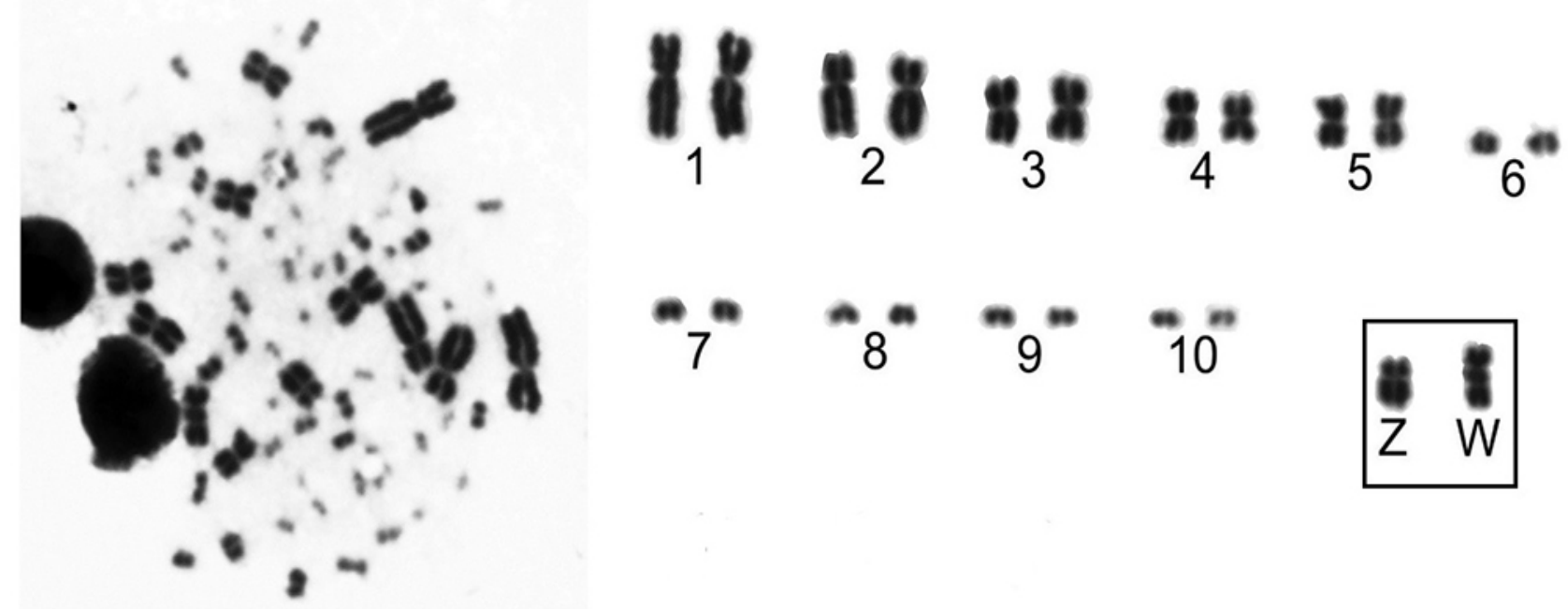

Figure 1 - Partial karyotype of Gallinula melanops (2n=80), stained with Giemsa showing only the macrochromosomes 1-10 and sex chromosomes ZW.

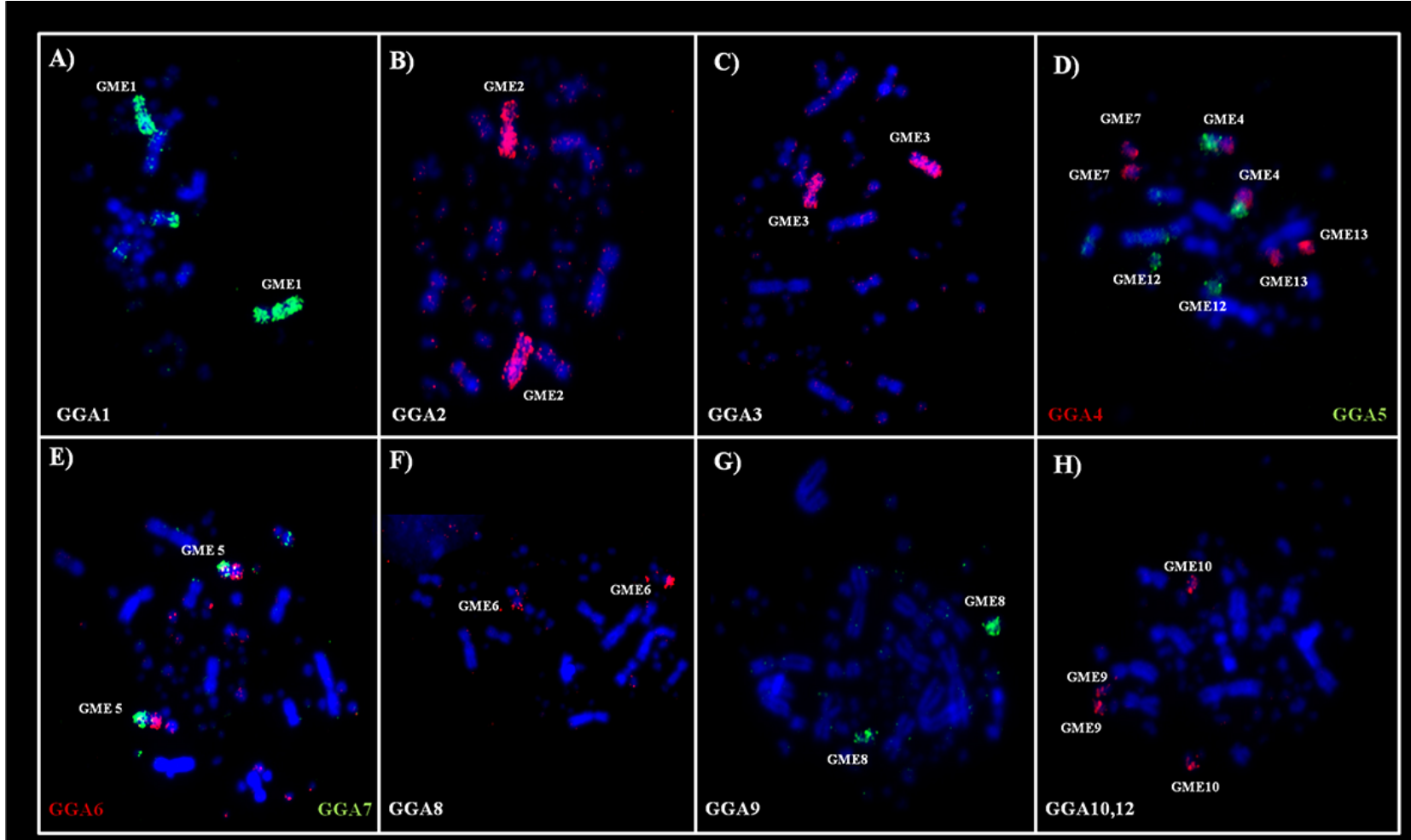

Figure 2 - FISH using whole-chromosome Gallus gallus probes on metaphases of Gallinula melanops. A-C and F-H are examples of conserved chromosomes, while D-E are examples of fusions and fissions. (D) GME4=GGA4 + GGA5 $\Rightarrow>$ fusion; GGA4 = GME4p + GME7+ GME13 $\Rightarrow>$ fission; GGA5 $=$ GME12 + GME4q $=>$ fission; and (E) evidence that GGA6 + GGA7 $=$ GME 5 = fusion. 
Table 1 - Correspondence between Gallus gallus (GGA) and Gallinula melanops (GME) chromosomes.

\begin{tabular}{|c|c|c|}
\hline Probes & Pair number & G.melanops \\
\hline GGA1 & 1 pair & GME1 \\
\hline GGA2 & 1 pair & GME2 \\
\hline GGA3 & 1 pair & GME3 \\
\hline GGA4 & 3 pair & GME $4 p, 7,13$ \\
\hline GGA5 & 2 pair & GME 4q, 12 \\
\hline GGA6 & 1 pair & GME 5p \\
\hline GGA7 & 1 pair & GME 5q \\
\hline GGA8 & 1 pair & GME 6 \\
\hline GGA9 & 1 pair & GME 8 \\
\hline GGA10-12 & 2 pairs & GME 9, 10 \\
\hline GGA11 & - & - \\
\hline GGA13 & 1 pair & GME 14 \\
\hline GGA14 & 1 pair & GME 15 \\
\hline
\end{tabular}

Table 2 - Correspondence between Gallus gallus BACs from microchromosome and Gallinula melanops microchromosomes.

\begin{tabular}{|c|c|c|}
\hline BAC names & G.gallus & G.melanops \\
\hline CH261-42P16 & $17 \mathrm{q}$ & $18 \mathrm{q}$ \\
\hline CH261-113A7 & $17 \mathrm{p}$ & $18 \mathrm{p}$ \\
\hline CH261-72B18 & $18 \mathrm{q}$ & $19 q$ \\
\hline CH261-60N6 & $18 \mathrm{p}$ & $19 p$ \\
\hline CH261-10F1 & $19 \mathrm{q}$ & $20 \mathrm{q}$ \\
\hline CH261-50H12 & $19 p$ & $20 p$ \\
\hline TGMCBA-250E3 & $20 \mathrm{q}$ & $21 \mathrm{q}$ \\
\hline TGMCBA-341F20 & $20 \mathrm{p}$ & $21 \mathrm{p}$ \\
\hline СH261-122K8 & $21 \mathrm{q}$ & $22 p$ \\
\hline CH261-83I20 & $21 p$ & $22 q$ \\
\hline CH261-18G17 & $22 q$ & $23 \mathrm{q}$ \\
\hline CH261-40J9 & $22 p$ & $23 p$ \\
\hline СH261-90K11 & $23 q$ & $24 q$ \\
\hline CH261-191G17 & $23 p$ & $24 p$ \\
\hline $\mathrm{CH} 261-65 \mathrm{O} 4$ & $24 q$ & $25 \mathrm{q}$ \\
\hline CH261-103F4 & $24 p$ & $25 \mathrm{q}$ \\
\hline СH261-127K7 & $25 \mathrm{q}$ & $26 \mathrm{q}$ \\
\hline СH261-59C21 & $25 p$ & $26 p$ \\
\hline CH261-170L23 & $26 \mathrm{q}$ & $27 \mathrm{q}$ \\
\hline CH261-186M13 & $26 \mathrm{p}$ & $27 p$ \\
\hline CH261-28L10 & $27 \mathrm{q}$ & $28 \mathrm{q}$ \\
\hline CH261-66M16 & $27 p$ & $28 \mathrm{p}$ \\
\hline CH261-64A15 & $28 \mathrm{q}$ & $29 \mathrm{q}$ \\
\hline CH261-72A10 & $28 \mathrm{p}$ & $29 p$ \\
\hline
\end{tabular}




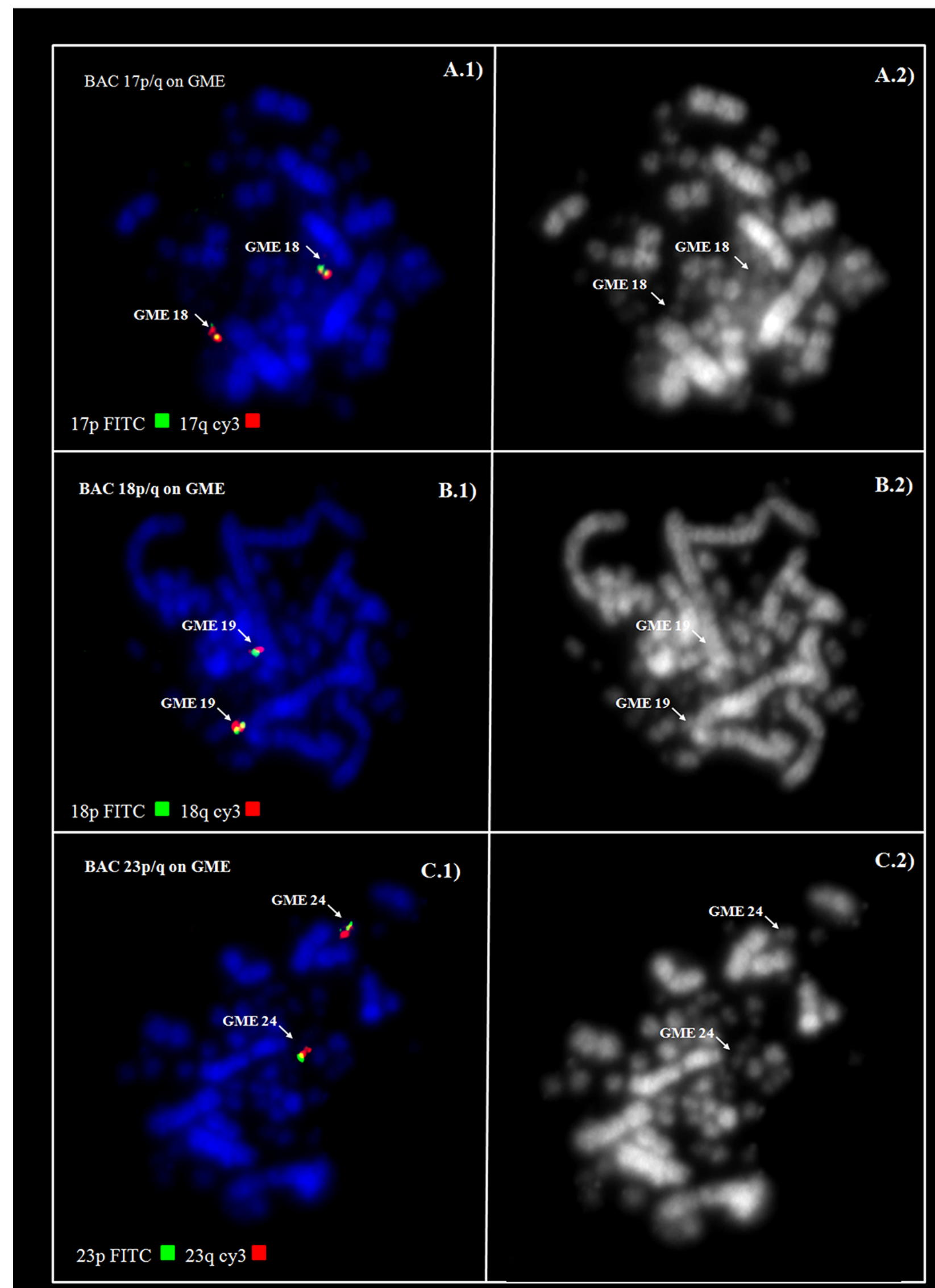

Figure 3 - Homology between chromosomes of Gallus gallus (GGA) and Gallinula melanops (GME) determined by FISH experiments using Chicken BACs from microchromosomes. Examples of conserved microchromosomes can be observed in: A1-A2- BACs 17p/q, B1-B2- BACs 18p/q, C1-C2BACs 23p/q. 

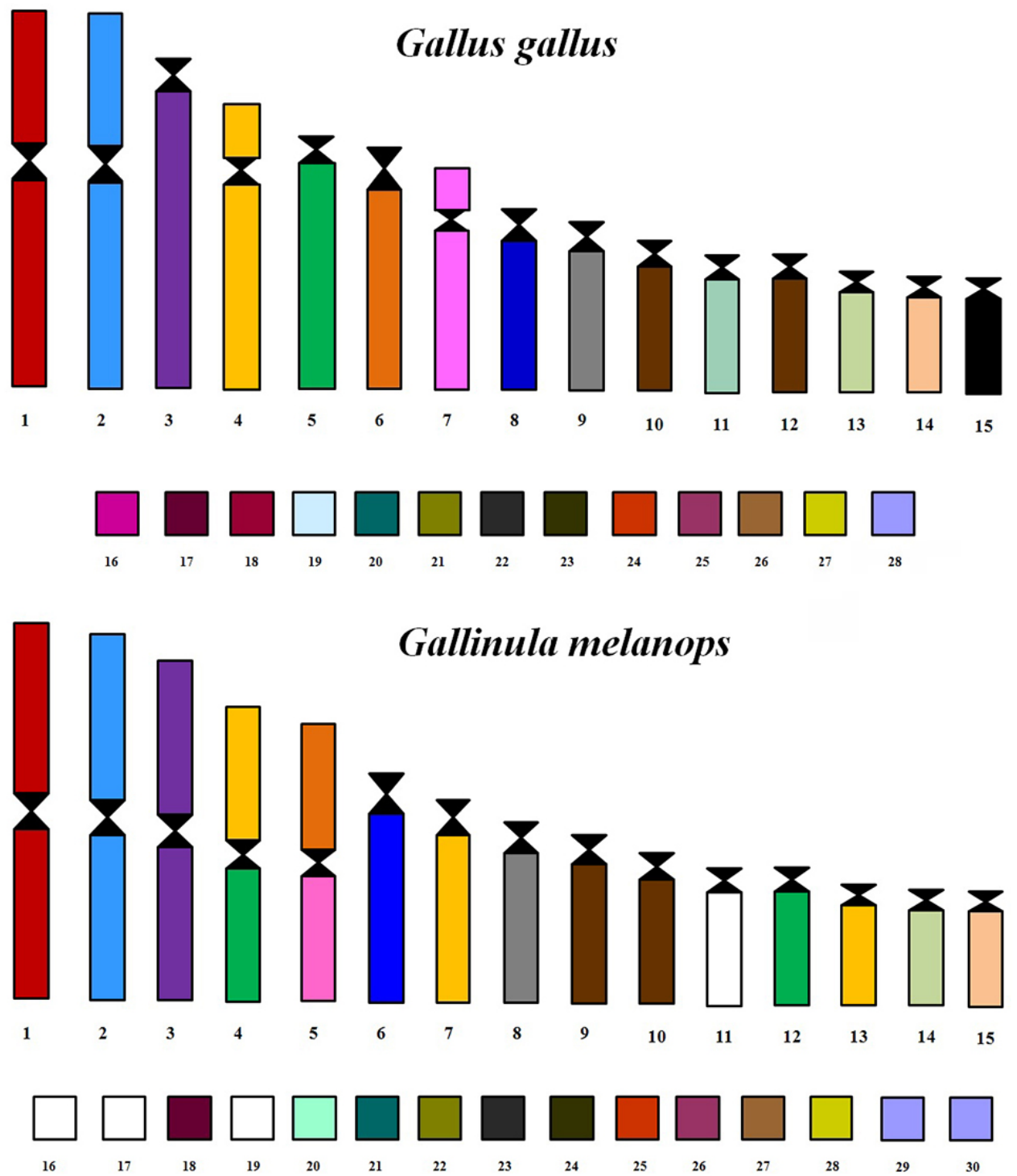

Figure 4 - Homology map between chromosomes of Gallus gallus and Gallinula melanops determined by FISH experiments with GGA painting probes (GGA1-14) and GGA BAC clones of microchromosomes from 17-28. A) reporting the colour guide to GGA painting probes and BAC clones, and B) reporting the homology between the chromosomes of these two species. Segments not hybridized are indicated in white. Chicken probe 11 did not work and BACs 15 and 16 were not used in this study. The BAC 20 of Zebra finch is represented by the BAC 20 of chicken.

\section{Discussion}

Gruiformes is among the avian orders with the least chromosomal information (Furo et al., 2015, 2020). Currently, karyological data obtained by classical cytogenetic methods are available for 30 out of a total of 189 species of Gruifromes. Among these species, only six species (belonging to four out of 33-40 genera) from the family Rallidae were cytogenetically investigated (Garcia et al., 2014; Gill et al., 2020): Fulica atra, $2 \mathrm{n}=92$; Gallinula chloropus, $2 \mathrm{n}=78$; Aramides cajaneus, $2 \mathrm{n}=78$; G. melanops, $2 \mathrm{n}=80 ;$ Porzana albicollis, $2 \mathrm{n}=72 ;$ Porphyiro porphyrio, 2n=80 (Giannoni and Giannoni, 1983; Hassan, 1998; Nanda et al., 2011; Gunski et al., 2019; Furo et al., 2020).

A comparison of chromosome morphology available for this family shows that generally the first six pairs are biarmed, while the remaining macrochromosomes are telocentrics, except in $P$. albicollis, which has a karyotype of only biarmed macrochromosomes (Table 3). Compared to other Gruiformes, which usually follow a chromosome pattern common to each family, there is great diversity in chromosomal morphology in Rallidae species, due to inversions, fusions and fissions, which play an important role in the karyotype evolution within this family (Furo et al., 2015).

Comparative chromosome painting data using G. gallus probes in Rallidae species are restricted to three genera: Fulica, Gallinula and Aramides (Nanda et al., 2011; Furo et al., 2020). The chromosome rearrangements found in this group involved chromosomes GGA4, GGA5, GGA6 and GGA7. In Fulica and Gallinula genera, the fission of GGA4 and GGA5, and fusions between GGA4/GGA5 and GGA6/GGA7 were observed in F. atra, G. chloropus (Nanda et al., 2011) and also in $G$. melanops $(2 \mathrm{n}=80)$, analyzed herein (Figure 4$)$. Probably, these rearrangements were already present in the common ancestor of Clade Fulica.

Furthermore, it was possible to confirm that each chicken or Zebra finch BAC was conserved as a distinct element in each microchromosome of G. melanops (Figure 3). 
Table 3 - Morphological classification of macrochromosomes of five species belonging to the Rallidae family. Legend: M- Metacentric; SM- Submetacentric; T- Telocentric.

\begin{tabular}{|c|c|c|c|c|c|c|c|c|c|c|c|}
\hline \multicolumn{12}{|c|}{ Chromosome morphology } \\
\hline \multirow[b]{2}{*}{ Species } & \multicolumn{10}{|c|}{ Chromosomes } & \multirow[b]{2}{*}{ Reference } \\
\hline & 1 & 2 & 3 & 4 & 5 & 6 & 7 & 8 & 9 & 10 & \\
\hline Fulica atra & M & SM & M & M & SM & M & $\mathrm{T}$ & $\mathrm{T}$ & $\mathrm{T}$ & $\mathrm{T}$ & Nanda et al., 2011 \\
\hline Gallinula chloropus & M & SM & M & M & SM & M & $\mathrm{T}$ & $\mathrm{T}$ & $\mathrm{T}$ & $\mathrm{T}$ & Nanda et al., 2011 \\
\hline Gallinula melanops & SM & SM & M & M & M & $\mathrm{T}$ & $\mathrm{T}$ & $\mathrm{T}$ & $\mathrm{T}$ & $\mathrm{T}$ & Gunski et al., 2019 \\
\hline Porzana albicollis & M & M & SM & SM & SM & M & M & M & M & M & Giannoni and Giannoni, 1983 \\
\hline Porphyrio porphyrio & M & M & M & M & M & SM & $\mathrm{SM}$ & A & A & A & Hassan, 1998 \\
\hline Aramides cajaneus & SM & SM & $\mathrm{T}$ & SM & M & M & $\mathrm{T}$ & M & $\mathrm{T}$ & $\mathrm{T}$ & Furo et al., 2020 \\
\hline
\end{tabular}

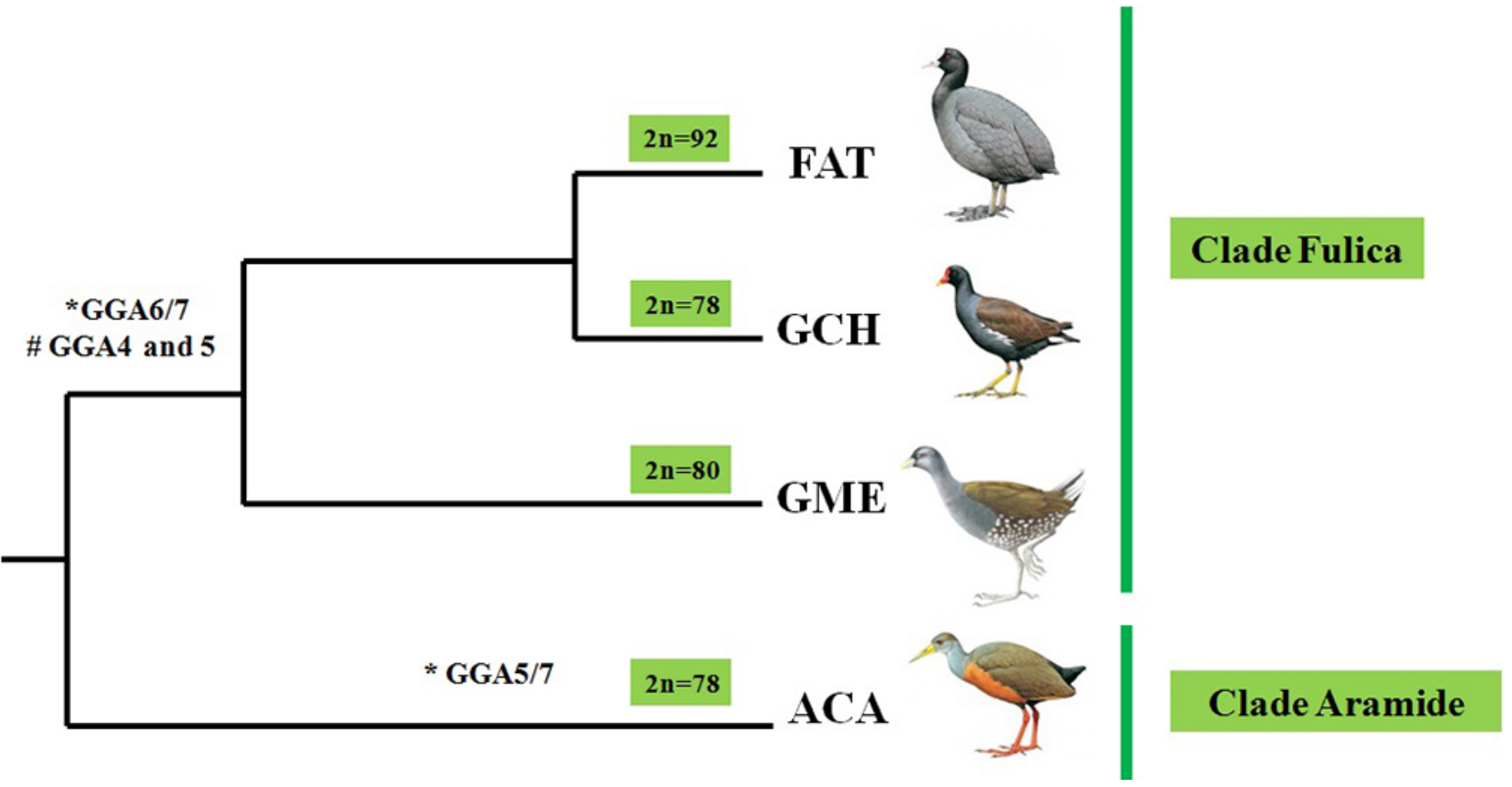

Figure 5 - An adaptation of the phylogenetic tree proposed by Garcia-R et al. (2014), plotted with FISH data from G.gallus probes (Legend: FAT- Fulica atra; GCH- Gallinula chloropus; ACA-Aramides cajaneus; GME- Gallinula melanops). (*) correspond fusion and (\#) fission.

Microchromosomes are highly conserved in bird karyotypes, with rearrangements involving these elements detected only is some orders, such as Psittaciformes and Falconiformes (O'Connor et al., 2019). Despite the conservation of microchromosomes in the karyotype of $G$. melanops, the increase in diploid number in $F$. atra $(2 \mathrm{n}=92)$ can be explained by extensive fission of microchromosomes.

According to Sangster et al. (2015), G. melanops should be included in the genus Porphyiro. However, the chromosome morphology data of $P$. porphyrio, the only species from this genus with a known karyotype, do not show many similarities with the karyotype of $G$. melanops. For example, in $G$. melanops the macrochromosomes 1-5 are biarmed and 6-10 are telocentrics, whereas in $P$. porphyrio chromosomes 1-7 are biarmed and 8-10 are acrocentrics (Table 3).

The phylogenetic relationships within the 'Clade Fulica' (genera Fulica, Gallinula and Porzana), based on mitochondrial DNA (mtDNA), suggest that this group is paraphyletic (Sangster et al., 2015). In the analysis based on mitochondrial and nuclear genes (Cytb, COI, 16S, FGB-7, $R A G-1)$, performed by Garcia et al. (2014), G. melanops
$(2 \mathrm{n}=80)$ was recovered as the sister clade to $G$. chloropus $(2 n=78)$. However, these species share the same chromosome rearrangements, which could indicate that their common ancestor would contain the fission into GGA4 and GGA5, aside from the association between GGA4/GGA5 and GGA6/GGA7.

Furthermore, other phylogenetic analyses using mtDNA recovered G. melanops in a more basal position within Clade Fulica (species of genera Fulica and Gallinula) (Garcia et al., 2014; Sangster et al., 2015), consistent with the chromosome painting data that indicate the karyotypic similarity between G. melanops and G. chloropus (Figure 5).

Additionally, the clade Aramides would be sister group to clade Fulica, despite the species A. cajaneus (Clade Aramides) not showing the fission into GGA4 and GGA5, or the fusions between GGA4/GGA5 and GGA6/GGA7 (Furo et al., 2020). Thus, the last common ancestor of these clades would have a karyotype similar to the putative avian ancestral karyotype (Furo et al., 2020).

In conclusion, the comparative chromosome painting reveals that $G$. melanops has a similar karyotype to $G$. chloropus and does not support the separation of these species into different 
genera. They are supported as sister species. Additionally, as in most birds studied so far, the microchromosomes are conserved as distinct pairs and do not take part in interchromosomal rearrangements (fusions or fissions). The results illustrate the value of comparative chromosome painting and BAC mapping in phylogenetic studies.

\section{Acknowledgements}

We are grateful to the staff of Laboratório de Diversidade Genética Animal from Universidade Federal do Pampa (Campus São Gabriel), CNPq (309699/2015-0), PROPESPUFPA and CAPES for technical and financial support. This research was partially funded by a grant to $\mathrm{EHCO}$ from $\mathrm{CNPq}$ (307382/2019-2) and to MAFS from the Wellcome Trust in support of the Cambridge Resource Centre for Comparative Genomics and by the Biotechnology and Biological Sciences Research Council (BB/K008161/1) to the University of Kent, also by UIDB/CVT/00772/2020 funded by the Fundação para Ciência e Tecnologia (FCT).

\section{Conflict of Interest}

The authors declare that they have no conflict of interest.

\section{Authors Contributions}

IOF and EHCO conceived and designed the experiments. IOF provided the first draft of the manuscript. AVG, RJG and RK collected the specimens and performed the cell culture. IOF and RK performed the FISH experiments. PCOB, MAFS, JCP were responsible for generating the chicken whole chromosome probes and DKG and REO'C were responsible for generating the BACs chicken from microchromosomes. English review and manuscript critical review MAFS, PCOB, AVG, RJG, DKG and REO'C. Funding acquisition EHCO, MAFS, AVG, RJG, JCP and DKG. EHCO and MAFS coordinated the research. All authors corrected, revised and discussed the data.

\section{References}

de Oliveira EHC, Tagliarini MM, Rissino JD, Pieczarka JC, Nagamachi CY, O'Brien PCM and Ferguson-Smith MA (2010) Reciprocal chromosome painting between white hawk (Leucopternis albicollis) and chicken reveals extensive fusions and fissions during karyotype evolution of Accipitridae (Aves, Falconiformes). Chromosome Res 18:349-355.

Del Hoyo J, Elliot A, Sargatal J and Cabot J (1996) Handbook of the birds of the world - Hoatzin to Ausk. Lynx Editions, Barcelona, vol. 3, $821 \mathrm{p}$.

Dobigny G, Ducroiz JF, Robinson TJ and Volobouev V (2004) Cytogenetics and cladistics. Syst Biol 53:470-484.

Fain MG, Krajewski C and Houde P (2007) Phylogeny of 'core Gruiformes' (Aves: Grues) and resolution of the LimpkinSungrebe problem. Mol Phylogenet Evol 43:515-529.

Furo IO, Kretschmer R, O'Brien PCM, Ferguson-Smith MA and de Oliveira, EHC (2015) Chromosomal diversity and karyotype evolution in South American macaws (Psittaciformes, Psittacidae). PLoS One 10:e0130157.

Furo IO, Kretschmer R, O’Brien PCM, Pereira J, Ferguson-Smith MA and Oliveira EHC (2020) Phylogenetic analysis and karyotype evolution in two species of core Gruiformes: Aramides cajaneus and Psophia viridis. Genes 11:307.
Furo IO, Kretschmer R, O’brien PCM, Pereira JC, Garnero ADV, Gunski RJ, Ferguson-Smith MA and de Oliveira EHC (2018) Chromosome painting in neotropical long- and short-tailed parrots (Aves, Psittaciformes): Phylogeny and proposal for a putative ancestral karyotype for Tribe Arini. Genes 9:491.

Garcia JC, Gibb GC and Trewick AS (2014) Deep global evolutionary radiation in birds: Diversification and trait evolution in the cosmopolitan bird family Rallidae. Mol Phylogenetics Evol 81:96-108.

Gill F, Donsker D and Rasmussen P (eds) (2020) IOC World Birds List (v.10.2), https://www.worldbirdnames.org

Giannoni ML and Giannoni MA (1983) Cytogenetic analysis of the species Porzana albicollis ('Saracura-sanã' or Sora). Rev Bras Genet 6:649-665.

Gunski RJ, Kretschmer R, de Souza MS, Furo IO, Barcellos AS, Costa AL, Cioffi MB, de Oliveira EHC and Garnero ADV (2019) Evolution of bird sex chromosomes narrated by repetitive sequences: Unusual W chromosome enlargement in Gallinula melanops (Aves: Gruiformes: Rallidae). Cytogenet Genome Res 158:152-159.

Hackett SJ, Kimball RT, Reddy S, Bowie RCK, Braun EL, Braun MJ, Chojnowski JL, Cox WA, Han KL, Harshman J et al. (2008) A phylogenomic study of birds reveals their evolutionary history. Science 320:1763-1768.

Hassan HA (1998) Karyological studies on six species of birds. Cytologia 63:349-363.

Jarvis ED, Mirabas S, Abrer AJ, Li B, Houde P, Li C, Ho SYW, Faircloth BC, Nabholz B and Howard J (2014) Whole-genome analyses resolve early branches in the tree of life of modern birds. Science 346:1320-1331.

Kretschmer R, Gunski RJ, Garnero ADV, Furo IO, O'Brien PCM, Ferguson-Smith MA and de Oliveira EHC (2014) Molecular cytogenetic characterization of multiple intrachromosomal rearrangements in two representatives of the genus Turdus (Turdidae, Passeriformes). PLoS One 9:e103338.

Kretschmer R, de Oliveira EHC, dos Santos MS, Furo IO, O'Brien PCM, Ferguson-Smith MA, Garnero ADV and Gunski RJ (2015) Chromosome mapping of the large elaenia (Elaenia spectabilis): Evidence for a cytogenetic signature for passeriform birds? Biol J Linn Soc 115:391-398.

Kretschmer R, Ferguson-Smith MA and de Oliveira EHC (2018a) Karyotype evolution in birds: From conventional staining to chromosome painting. Genes 9:181.

Kretschmer R, Furo IO, Gunski RJ, Garnero ADV, Pereira J, O’Brien PCM, Ferguson-Smith MA, de Oliveira EHC and Freitas T (2018b) Comparative chromosome painting in Columbidae (Columbiformes) reinforces divergence in Passerea and Columbea. Chromosome Res 26:211-223.

Kretschmer R, Furo IO, Cioffi MDB, Gunski RJ, Garnero ADV, O'Brien PCM, Ferguson-Smith MA, Freitas TRO and de Oliveira EHC (2020) Extensive chromosomal fissions and repetitive DNA accumulation shaped the atypical karyotypes of two Ramphastidae (Aves: Piciformes) species. Biol J Linn Soc 130:839-849.

Lithgow PE, O’Connor R, Smith D, Fonseka G, Mutery AA, Rathje C, Frodsham R, O'Brien P, Kasai F, Ferguson-Smith MA et al (2014) Novel tools for characterizing inter and intra chromosomal rearrangements in avian microchromosomes. Chromosome Res 22:85-97.

Livezey BC (1998) A phylogenetic analysis of the Gruiformes (Aves) based on morphological characters, with an emphasis on the rails (Rallidae). Philos Trans R Soc Lond B 353:2077-2151.

Nanda I, Benisch P, Fetting D, Haaf T and Schmid M (2011) Synteny conservation of chicken macrochromosomes 1-10 in different avian lineages revealed by cross-Species chromosome painting. Cytogenet Genome Res 132:165-181. 
Nie W, O’Brien PCM, Ng BL, Fu B, Volobouev V, Carter NP, Ferguson-Smith MA and Yang F (2009) Avian comparative genomics: reciprocal chromosome painting between domestic chicken (Gallus gallus) and the stone curlew (Burhinus oedicnemus, Charadriiformes) - an atypical species with low diploid number. Chromosome Res 17:99-113.

Nie W, O'Brien PCM, Fu B, Wang J, Su W, He K, Bed'Hom $\mathrm{B}$, Volobouev V, Ferguson-Smith MA, Dobigny G et al. (2015) Multidirectional chromosome painting substantiates the occurrence of extensive genomic reshuffling within Accipitriformes. BMC Evol Biol 15:205.

O'Connor RE, Kiazin L, Skinner B, Fonseka G, Joseph S, Jennings R, Larkin DM and Griffin DK (2019) Patterns of microchromosome organization remain highly conserved throughout avian evolution. Chromosoma 128:21-29.

Prum RO, Berv JS, Dornburg A, Field DJ, Townsend JP, Lemmon EM and Lemmon AR (2015) A comprehensive phylogeny of birds (Aves) using targeted next generation DNA sequencing. Nature 526:569-573.

Rodrigues BS, Kretschmer R, Gunski RJ, Garnero ADV, O'Brien PCM, Ferguson-Smith MA and de Oliveira EHC (2018) Chromosome painting in Tyrant flycatchers confirms a set of inversions shared by Oscines and Suboscines (Aves, Passeriformes). Cytogenet Genome Res 53:205-212.

Sangster G, García-R JC and Trewick SA (2015) A new genus for the Lesser Moorhen Gallinula angulata Sundevall, 1850 (Aves, Rallidae). Eur J Taxon 153:1-8.
Sasaki M, Ikeuchi T and Maino S (1968) A feather pulp culture for avian chromosomes with notes on the chromosomes of the peafowl and the ostrich. Experientia 24:1923-1929.

Seligmann ICA, Furo IO, dos Santos MS, Tagliarini MM, Araujo CCD, O’Brien PCM, Ferguson-Smith MA and de Oliveira EHC (2019) Comparative chromosome painting in two Brazilian stork species with different diploid numbers. Cytogenet Genome Res 159:32-38.

Taylor B and van Perlo B (1998) Rails: A guide to the rails, crakes, gallinules and coots of the world. Pica Press, Sussex.

Taylor PB (1996) Family Rallidae (Rails, Gallinules and Coots). In: del Hoyo J, Elliot A and Sargatal J (eds). Handbook of the Birds of World - Hoatzin to Auks. Lynx Edicions, Barcelona, vol. 3, pp 108-209.

Telenius H, Pelmear AH, Tunnacliffe A, Carter NP, Behmel A, Ferguson-Smith MA, Nordenskjöld M, Pfragner R and Ponder BA (1992) Cytogenetic analysis by chromosome painting using DOP-PCR amplified flow-sorted chromosomes. Genes Chromosomes Cancer 4:257-263.

Wetmore A (1960) A classification for the birds of the world. Smithsonian Institution, Washington, vol. 139, 45 p.

Associate Editor: Maria Jose de J.Silva

License information: This is an open-access article distributed under the terms of the Creative Commons Attribution License (type CC-BY), which permits unrestricted use, distribution and reproduction in any medium, provided the original article is properly cited. 